\title{
Personal Protective Equipment (PPE): A Solution to Occupational Hazard or An Occupational Hazard Itself?
}

\author{
Neha Verma* and Meenu G Singla \\ Department of Conservative Dentistry and Endodontics, Sudha Rustagi College of Dental Sciences and Research, Faridabad (Haryana), \\ India
}

*Corresponding author: Neha Verma, Department of Conservative Dentistry and Endodontics, Sudha Rustagi College of Dental

Sciences and Research, Faridabad (Haryana), India

\begin{abstract}
Personal protective equipment (PPE) has become an important and much talked about subject during the current coronavirus pandemic. Since the dental profession predominantly involves exposure of the dentist to various body fluids such as saliva, blood etc., the use of PPE is imperative for infection control in dental practice. However, even though protection is successfully achieved by PPE, such high level of protection is likely to entail additional workloads and occupational hazards on healthcare professionals including dentists. The present review aims to throw light on the occupational hazards that can occur and some tips to alleviate the discomfort following the use of PPE. These methods are relatively simple to follow while maintaining normal healthcare routine.
\end{abstract}

Keywords: Personal Protective Equipment (PPE); Occupational Hazard; Dentistry; COVID-19

\section{Introduction}

Occupational hazard refers to a risk or danger as a consequence of the nature or working condition of a particular job [1]. Dentists while carrying out their professional work are exposed to various occupational hazards; one of them is getting infected from the patient [2]. These days the world is suffering from novel coronavirus (2019-nCoV), the seventh member of the family "coronaviruses" which infects humans [3]. Dentists also are at tremendous risks of acquiring this infection from their patient. COVID-19 transmission in dental settings occurs through four major routes:

a. Direct exposure to respiratory secretions containing droplets, saliva, blood or other patient materials.

b. Direct inhalation of suspended airborne viruses.

c. Indirect contact with contaminated surfaces and/or instruments.

d. Mucosal (nasal, oral and conjunctival) contact with infection-containing droplets and aerosols that are propelled by coughing and talking without a mask [4].
Due to the Specificity of dental procedures which include dealing with head and neck region, especially performing aerosol generating procedures in the oral cavity, the risk of COVID-19 transmissions in dental professionals is very high [5]. In spite of these vulnerabilities of COVID-19 transmissions which expose one to endanger, the dental professionals cannot refrain from providing care and serving community $[4,5]$. Thus, it is of utmost importance to incorporate maximum precautionary measures including donning PPE in order to prevent transmission and spread of infection among dental professionals. PPE includes surgical scrub suit, eye protection, different types of masks, fluid resistant gown, disposable apron, gloves, head cap, face shield, shoe cover etc., which is designed to protect the skin and the mucous membranes of the eyes, nose and mouth of dental healthcare personnel from exposure to contaminated blood, saliva, aerosols or other potentially infectious material[6].

But dental professionals are facing many problems while using these PPE for example wearing eye protection, face shields and face mask simultaneously results in fogging and glaring which 
ultimately leads to restricted vision. Long term utilisation of N95 mask results in hypoxemia and hypercapnia eventually causes headache. Scar on the nasal bridge and cheeks may be caused by the excessive pressure of tying mask tightly and squeezing the metal clip hard. Also, wearing N95 mask along with heavy respirator can further cause difficulty in communication with the patient. Wearing multiple layers of PPE results in body's inability to cool down adequately known as heat stress which causes dehydration, breathing difficulty, profuse sweating, dizziness, dry mouth, dark urine, exhaustion. Apart from these other problems are skin irritation, runny/blocked/stuffy nose, dry/itchy/watery eyes, sneeze, cough etc [7-9].

Various measures can be taken by the dental professionals to alleviate the discomfort while wearing PPE which can be as follow:

a. Prefer ventilated environment for dental procedures.

b. Apply moisturiser at least 30 minutes before donning PPE.

c. PPE should be of proper fitting.

d. Pay attention to the material of clothing worn inside. Select breathable, light-coloured, moisture-wicking fabrics for surgical scrub suits, gowns and surgical caps or take it one step further to try cooling vests which can be fitted with ice pack.

e. As the Surgical cap can retain heat. One can leave his/her hair slightly damp under the cap to reduce the heat or choose a slightly larger size to allow heat to escape.

f. Face masks with ties should be preferred. Buttons on surgical caps can reduce strain on ears from mask ear loops. Ear savers are another great product to reduce the same.

g. To mitigate pressure for minimising the risk of pressure damage to the skin, barrier dressing tapes and liquid barrier film (which is easy to apply, dries only in 30 seconds and forms an invisible barrier for up to 72 hours even with normal washing) can be used under masks and goggles.

h. Sign/body languages or notepad can be helpful in avoiding communication difficulties with patients while wearing multiple layer of PPE.

i. Try not to wear PPE, especially FFP3 masks for more than two hours at a time.

j. Schedule in-between breaks.

k. To avoid eye problems, Eyes should be washed with cool water. Over-the-counter eye drops can also be helpful in soothing dry/itchy/watery/irritated eyes.

l. Drink enough fluids preferably water during the parts of the day when most convenient to do so. m. Avoid energy/sports/caffeinated/aerated/sugary/ alcoholic drinks which claims to support or enhance hydration. But can have negative impact on the kidneys due to very low $\mathrm{pH}$, high titratable acidity levels, high sodium content which can result in dehydration on excessive intake.

n. Fruit-infused water, water with a slice of lemon, Iced herbal teas, yogurt offer a refreshing alternative.

o. Try a drop of peppermint oil, cooling towels and cooling gel packs from the freezer. When these placed on pulse points such as neck, temples and wrists can reduce body heat quickly and create a cooling sensation during a break, setting dental professional up for the next patient with a cooler body temperature.

p. Practise various breathing exercises for increasing the lungs capacity [9-12].

\section{Conclusion}

It is important for dentists to know the best practices for control of risks while performing dental procedures. The use of Personal protective equipment (PPE) does reduce rates of disease transmission. However, it does pose health hazards to practicing dentists which should also be taken into consideration. We should take certain measures to counteract the side effects of Personal protective equipment (PPE) which are relatively simple to follow and easy to apply, while maintaining normal healthcare routine.

\section{References}

1. Chopra Ss, Pandey Ss (2007) Occupational Hazards Among Dental Surgeons. Mjafi 63: 23-25.

2. Reddy KS, Majumder DSP, Doshi D, Kulkarni S, Reddy S, et al. (2017) Occupational hazards in dentistry. J Res Adv Dent 6(2): 110-122.

3. Zhou P (2020) A pneumonia outbreak associated with a new coronavirus of probable bat origin. Nature 579: 270-273.

4. Peng X, Xu X, Li Y, Cheng L, Zhou X (2020) Transmission routes of 2019$\mathrm{nCoV}$ and controls in dental practice. Int J Oral Sci 12(1): p.9.

5. Fallahi HR, Keyhan So, Zandian D, Kim SG, Cheshmi B (2020) Being a front-line dentist during the Covid-19 pandemic: a literature review. Maxillofac Plast Reconstr Surg. 42(1): pp.12.

6. Amato A, Caggiano M, Amato M, Moccia G, Capunzo M, et al. (2020) Infection control in dental practice during the COVID-19 pandemic. International journal of environmental research and public health 17(13): pp.4769.

7. Loibner M, Hagauer S, Schwantzer G, Berghold A, Zatloukal K (2019) Limiting factors for wearing personal protective equipment (PPE) in a health care environment evaluated in a randomised study. PLoS ONE 14(1): e0210775.

8. Hu K, Fan J, Li X, Gou X, Li X, et al. (2020) The adverse skin reactions of health care workers using personal protective equipment for COVID-19. Medicine 99(24): e20603.

9. Honda H, Iwata K (2016) Personal protective equipment and improving compliance among healthcare workers in high-risk settings. Current opinion in infectious diseases 29(4): 400-406. 
10. Anne Nugent Guignon (2020) Are you more than just thirsty? The 12. Side effects of PPE: Tips to relieve physical and emotional. dangerous impacts of dehydration.

11. Erin Dean (2020) COVID-19: how to avoid skin damage while wearing PPE.

\section{(c) (†) \\ This work is licensed under Creative Commons Attribution 4.0 License}

To Submit Your Article Click Here:

Submit Article

DOI: 10.32474/RRHOAJ.2020.05.000225
Citation: Neha Verma, Meenu G Singla. Personal Protective Equipment (PPE): A Solution to Occupational Hazard or An Occupational Hazard Itself?. Res \& Rev Health Care Open Acc J 5(5)- 2020. RRHOAJ.MS.ID.000225. DOI: 10.32474/RRHOAJ.2020.05.000225. 